\title{
Reaction-Diffusion Stochastic Lattice Model for a Predator-Prey System
}

\author{
Áttila L. Rodrigues and Tânia Tomé \\ Instituto de Física \\ Universidade de São Paulo \\ Caixa postal 66318 \\ 05315-970 São Paulo- SP, Brazil
}

Received on, 17 December, 2007

\begin{abstract}
We have the purpose of analyzing the effect of explicit diffusion processes in a predator-prey stochastic lattice model. More precisely we wish to investigate the possible effects due to diffusion upon the thresholds of coexistence of species, i. e., the possible changes in the transition between the active state and the absorbing state devoid of predators. To accomplish this task we have performed time dependent simulations and dynamic mean-field approximations. Our results indicate that the diffusive process can enhance the species coexistence.
\end{abstract}

Keywords: Stochastic models; Predator-prey systems; Reaction-diffusion systems; Mean-field approach; Numerical simulation

\section{INTRODUCTION}

Computational physics descriptions and the usual analysis of numerical simulation data provided by the statistical mechanics can give worthy contributions to the study of population biology problems. A basic, but not trivial, issue of population biology, is the one concerned to the dynamics of a predator-prey system. This problem has been considered from the theoretical point of view since Lotka who in 1920 [1], [2], [3], followed by Volterra [4], has introduced the first model (to be called here LV model) able to elucidate the main features of species interactions and populational cycles in predatorprey systems. After that, the LV model has been modified to contemplate other important features of these systems and we may say that most of the theoretical approaches presently used to describe problems of the population biology have some basis on the LV model. Among those descriptions we point out the stochastic lattice gas models approach [5], [6], which will concern us in the present article. These models are able to provide spatial patterns of species distribution which can be coupled to local time oscillations as well as other important features of predator-prey systems [7], [8], [9], [10].

Doubtlessly a mark in the understanding of predator-prey interactions, and their coexistence in space distributed systems, is the experiment on predation performed by Huffaker in 1958 [11]. He has verified that an heterogeneous habitat can be crucial to maintain the coexistence of species for a long period of time. Experiments performed in homogeneous habitat have shown that predators eat prey very quickly, prey get extinct and then predators die of starvation. These experimental results have evidenced that the coexistence of species for a long period of time can be directly related to a heterogeneous spatial distribution of the individuals of each species. Particularly, this feature should be taken into account in the modelling of predator-prey systems which population sizes can attain very small values, or when populations loose contact and become isolated during some period of time. That is, when the species populations are spatially distributed.

Much of the theoretical developments to describe the rôle of space in population biology problems were, in a certain way, motivated by the Huffaker's experiments. We detach two relevant stochastic approaches which take into account the localization of species individuals and their local interactions: (i) the stochastic lattice gas models, also called interacting particle systems [7], [8]; (ii) the metapopulation approaches and patch models [12], [13], [14], [15]. In the last decades, both approaches have been largely used to analyze, at different levels of description, the different features of population biology problems. Several investigations have been developed in this issue and here we just cite some of them [7]-[10], [12]-[41].

In the present article we are concerned on the effect of diffusive processes in predator-prey systems described by the stochastic lattice model introduced by Satulovsky and Tomé [9]. However, before entering into the details of our stochastic dynamic analysis, we will briefly expose, in what follows, the main features of the LV model, as well as related models; patch models and spatial models for predator-prey systems are also exposed. This is not a review on theoretical approaches of population biology, but a very basic, brief and simple explanation of some of the features of some theoretical approaches. Our purpose is just to clarify the issue treated in the present article.

\section{A. Lotka-Volterra model and related models}

The definition and description of the dynamics of predatorprey system consists in one of the fundamental problems of the population biology. One considers two species, predator and prey, sharing the same habitat. To understand the dynamics of coexistence of both populations it is necessary to establish the main characteristics of their interaction. We depart from the LV model for a predator-prey system which is defined by a set of two ordinary differential equations for prey size population, denoted by $H$, and predators size population, denoted by $P$. This description is based on the law of mass action and the differential equations can be written in the following form

$$
\frac{d H}{d t}=r_{1} H-r_{2} H P
$$




$$
\frac{d P}{d t}=r_{2} H P-r_{3} P
$$

where $r_{1}, r_{2}$ and $r_{3}$ are nonnegative constants related to the processes considered in the LV model. The process of proliferation of prey is proportional to the prey number, which is expressed by the term $\left(r_{1} H\right)$ in Eq. (1). Thus, in the absence of predators the prey population size increases exponentially in time. This result comes from the assumption that there is an unlimited amount of resources and space for prey proliferation. The predator-prey interaction, the most relevant and crucial process, is expressed as the product of predators number and the prey number (term $\left(r_{2} H P\right)$ in Eqs. (1) and (2)). According to this autocatalytic process, predators increase their number just when consuming prey and there is just one prey species available for them. Finally, predators size population decreases according to a spontaneous process which occur at rate $r_{3}$. It is worth to note that it is implicitly assumed in the LV model that predators and prey populations are very well mixed in an homogeneous habitat. These are usual presupposes when one uses the law of mass action, as it was used to derive all terms of Eqs. (1) and (2). In this case the predatorprey system can be described at any instant of time $t$ only by their size populations, that is, by $(H(t), P(t))$.

The trivial solution of Eqs. (1) and (2) is unstable and the non-trivial one is a center for which any perturbation around is periodic. Once an initial condition is fixed we can say that both population sizes oscillate in time with the same period, out-of-phase and a peak in the prey number is always followed by a peak in the predators number. These are relevant features of the population cycles encountered in experiments on predation [11] and in nature. In this case, a famous example consists on the long term data related to the population cycles of the snowshoe hare and the lynx of the Canadian boreal forest [43], [44], [45], [46], [47], [48], [49].

However, the LV model displays infinitely many periodic orbits in the $P-H$ phase plane and these are related to different initial conditions [44]. In this respect the LV model fails in describing biologic populations, since it is not expected that a small change on the initial assumptions will lead to different population cycles [48]. In order to answer for the stability of a predator-prey system important modifications in the LV model were performed. One of them consists in taking into account the fact that there exists a maximum prey population size that the system can support since the amount of food for prey proliferation is not unlimited. This feature can be expressed by the consideration of a logistic term to describe prey proliferation. So that the first term in Eq. (1) is replaced by $r_{1} H(1-H / C)$, where $C$ is a constant related to the maximum prey number, and the constant $r_{1}$ is the prey reproduction rate (in the absence of predators). The resulting modified LV model predicts stable nontrivial solutions which are attained by damped oscillations of the population sizes. Another relevant modification of the LV model that has been considered concerns to the response of predators to prey population size changes, the so called functional responses $f(H)$ [48]. One consider the term related to predator-prey interaction in Eqs. (1) and (2) written as $\left(r_{2} f(H) P\right)$. In the original LV model $f(H)$ is linear; in general it is stated according to the biolog- ical effect to be described. Interesting functional responses where considered by Holling and Tanner [47]. Their models, for specific sets of parameters, are able to predict species coexistence which can be stable or related to population cycles. These sets of parameters and the different models seem to describe qualitatively some predator-prey systems observed in nature [50].

\section{B. Patch models}

A more sophisticated approach to describe the dynamics of a predator-prey system can be attained considering patch models and the metapopulation models approaches [13], [14]. This description was primordially considered by Levins in 1969 [42] to analyze a single species system whose individuals can proliferate and die at given rates. The main feature captured in the Levins approach relies in the fact that it is capable to recognize the necessity of taking into account the space available for the species proliferation. In the metapopulation approach it is considered that the population can be subdivided in subpopulations which live in interconnected habitat patches. Instead of treating the dynamics of the entire population sizes, one treats the dynamics of the fraction of patches occupied by species individuals. The most simple patch formulation, as stated by Levins, consists of an ordinary differential equation for the fraction of patches occupied by the species individuals; this equations is build up on the basis of the law of mass action. A natural extension of Levins approach for a two-species system, as a predator-prey system, could be accomplished considering three types of patches: empty patches, patches occupied by prey and patches occupied by predators. The dynamics of such a model can be stated as follows: prey can proliferate in empty patches with a given rate $a$, predators can proliferate in prey patches at rate $b$, and can die, leaving an empty patch, with a given rate $c$. In this context a simple description, which takes into account the LV model mechanism of interaction between the two species, may be given by,

$$
\begin{gathered}
\frac{d x}{d t}=a x(1-x-y)-b x y \\
\frac{d y}{d t}=b x y-c y,
\end{gathered}
$$

where $x$ is the fraction of patches occupied by prey, $y$ the fraction of patches occupied by predators and $(1-x-y)$ is the fraction of empty patches. This set of equations can be regarded as a special case of a patch model analyzed by Hastings [15]. We remark that, as we will see in section III, the same equations are also provided by the simple mean-field approximation of a lattice gas model for a predator-prey system. The first term in Eq. (3), $a x(1-x-y)$, is a logistic term which takes into account the space available for prey proliferation. The fraction of patches occupied by prey (predators) can be seen as the density of prey (predators). The structure of the 
set of Eqs. (3) and (4) differ from the structure of the set of Eqs. (1) and (2) just by the logistic term. This patch model display nontrivial stable stationary solutions corresponding to stable coexistence of prey and predators.

The metapopulation approach has been developed to include local stochastic dynamics and is nowadays largely used to model ecological problems. One of the issues investigated in the scope of this approach concerns to the dynamics of species distributed over fragmented habitats. A relevant problem treated in this case concerns the relationship between the fragmentation of the habitat and the conservation of species and biodiversity [14], [29], [37].

\section{Stochastic lattice models}

Theoretical descriptions based on the assumptions of the LV modified models, as the ones above described, can be adequate if we want to describe the dynamics of species populations which are mixed and dispersed over an homogeneous space. In this case the descriptive level of the population dynamic problem is such that the spatial structure is not relevant. However, under certain circumstances, one needs a theoretical approach which takes into account the spatial structure of the habitat and the localization of the individuals of each species. So, we need to establish the level of description needed and desired to describe a given population biology problem. This problem has been very well placed by Durrett and Levin [7]. They have treated the dynamics of a biological population problem by considering different levels of description and, consequently, different theoretical approaches.

That study gives particular attention to the approach based on stochastic lattice gas models, also called interacting particle systems $[5,6]$, which has been largely exploited, in the last years. A multiplicity of works have evidenced the importance of spatial structured models to describe ecological problems [7], [8]. The approach of the stochastic lattice gas models is based on Markovian processes defined on discrete spaces, which are able to take into account the localization of discrete individuals of a given population. Such a description allows us to treat the important features for the determination of the thresholds of species coexistence, and spatial patterns of distribution of the species individuals, by considering simple models [10], [27], [32]. Usually the spatial stochastic structured models are studied by means of computational simulations and dynamic mean-field approximations.

One of the first systematic statistical mechanics analysis of a very simple stochastic lattice model for a predator-prey system was performed by Satulovsky and Tomé in 1994 [9]. The model, to be called ST model, is able to exhibit local time oscillations of species populations and stable coexistence. Other deeper investigations on this model and similar models followed [10], [27], [28], [34], [38]. The ST model has a phase diagram displaying an active phase, where there is species coexistence with local time oscillations and coexistence with stationary densities; it also exhibits an absorbing phase which correspond to the extinction of one of the species. A version of the ST model with synchronous updating, a PCA, was considered by de Carvalho, Tomé and Arashiro [10, 31, 33, 38]. In this case it has been shown [38] that the whole transition line between the active and absorbing states is a line of critical points which belongs in the directed percolation universality class but one point belonging in the dynamic percolation universality class [16].

It is worth to note that mean-field approaches for spatial models, as the ST model [9] and its synchronous version [40] are able to provide species coexistence with self-sustained coupled time oscillations which are attained from a regime of stable coexistence by a Hopf bifurcation. The oscillations are stable against any perturbation on the initial conditions and they have a very well defined amplitude and period. The latter is the same for the prey density time series and predators density time series, and an abundance of prey is always followed by an abundance of predators. However, if the spatial structure of these models is explicitly considered, for example, when one performs Monte Carlo simulations on square lattices, it can be verified that the amplitude of oscillations decays as the system size increases; and, it vanishes in the thermodynamic limit [9], [33]. Therefore, oscillations are not globally synchronized, but remain synchronized in finite and small regions of the space; these are called local oscillations and have the property of being coupled to heterogeneous and interesting spatial patterns of species distribution.

The same behavior is observed for other spatial models [8]: coexistence with time oscillations resulting from Hopf bifurcations provided by mean-field approaches, and local oscillations with pattern formation when the spatial structure of the model is indeed considered. From these results we could infer that population cycles observed in nature and in experiments may be synchronized at subregions of the habitat. However, we must be careful with these results, since, usually, the predator-prey lattice models are still to crude to be directly compared with data obtained from experiments and from collected data for population cycles in nature. We can drawn some analogies and try to capture the fundamental properties of prey-predator systems. For example, the ST model [9], exhibits for a given range of the model parameters, pronounceable predator and prey oscillations [10] which seem to be qualitatively similar to the hare-linx population cycles. However, until now, it was not included in this model the inherent features, as intrinsic population growth rates, which allows us to make direct relationships between the theoretical description and the observed population cycles.

\section{Scope of this article}

In the present paper we focus on the dynamics of a predatorprey system and analyze the relevance of modelling the explicit movement of individuals. We depart from the stochastic lattice model for a predator-prey system [9, 25], the ST model, and include local stochastic processes of diffusion. Our analysis is performed by means of time dependent simulations $[5,51]$ which is one of the appropriated simulation techniques to investigate nonequilibrium phase transitions to 
an absorbing state. We also perform mean-field approximations. In section II we define the predator-prey stochastic model with diffusion. In section III we analyze the model by means of simple and pair mean-field approximations. In section IV we show our computational simulation results and in section $\mathrm{V}$ we present a discussion of our results.

\section{PREDATOR-PREY STOCHASTIC LATTICE MODEL WITH DIFFUSION}

We model a predator-prey system by a Markovian stochastic dynamics defined in a discrete space where individuals of each species are localized. The dynamics is asynchronous and comprehends a set of subprocesses which mimic the reaction processes of birth, death and predation and the explicit movement of individuals of each species by diffusive local processes.

We consider a regular lattice which represents the habitat where individuals can survive and interact with each other. For each site $i$ on the lattice, $i=1,2, \ldots, N$, we associate a stochastic discrete variable $\eta_{i}$ which can take the values: 1 , if site $i$ is occupied by a prey individual; 2 , if site $i$ is occupied by a predator and 0 , if site $i$ is empty. Each site can be occupied by at most one individual. The stochastic dynamics is defined over the configurational space spanned by the stochastic vector, $\eta=\left(\eta_{1}, \eta_{2}, \ldots, \eta_{N}\right)$, the microscopic state. The time evolution of the probability $P(\eta, t)$ associated to the stochastic vector $\eta$ at time $t$ is given by the master equation,

$$
\frac{d}{d} P(\eta, t)=\sum_{\eta^{\prime}}\left\{W\left(\eta \mid \eta^{\prime}\right) P\left(\eta^{\prime}, t\right)-W\left(\eta \mid \eta^{\prime}\right) P\left(\eta^{\prime}, t\right)\right\},
$$

where $W\left(\eta \mid \eta^{\prime}\right)=w\left(\eta \mid \eta^{\prime}\right) / \tau$ is the conditional transition rate, from $\eta^{\prime}=\left(\eta_{1}^{\prime}, \eta_{2}^{\prime}, \ldots, \eta_{N}^{\prime}\right)$ to $\eta$, and $w\left(\eta \mid \eta^{\prime}\right)$ is defined by the expression,

$$
w\left(\eta \mid \eta^{\prime}\right)=d w_{\text {diff }}\left(\eta \mid \eta^{\prime}\right)+(1-d) w_{\text {react }}\left(\eta \mid \eta^{\prime}\right),
$$

where $d$ is the probability of diffusion, $w_{\text {diff }}\left(\eta \mid \eta^{\prime}\right)$ denotes the conditional probability transition associated to the diffusive processes and $w_{\text {react }}\left(\eta \mid \eta^{\prime}\right)$ denotes the conditional probability transition associated to the reactive processes. In what follows we define each of these processes.

i) Diffusion. The possible diffusion processes are:

$$
\begin{gathered}
X+Z \rightleftarrows Z+X, \\
Y+Z \rightleftarrows Z+Y, \\
X+Y \rightleftarrows Y+X,
\end{gathered}
$$

where $X$ denotes prey $\left(\eta_{i}=1\right), Y$ denotes predators $\left(\eta_{i}=2\right)$ and $Z$ denotes a vacant site $\left(\eta_{i}=0\right)$, that is, food supply for prey and therefore a source for prey proliferation. We chose at random a pair of nearest neighbors sites $i$ and $j$. After that we exchange the states of these two sites with probability $d$. We define the transition probability associated to this process, which was denoted in expression (6) by $w_{\text {diff }}\left(\eta \mid \eta^{\prime}\right)$, as follows:

$$
w_{\text {diff }}(10 \mid 01)=\delta\left(\eta_{i}, 1\right) \delta\left(\eta_{j}, 0\right)+\delta\left(\eta_{i}, 0\right) \delta\left(\eta_{j}, 1\right)
$$

which is related to diffusion of prey in empty sites;

$$
w_{\text {diff }}(12 \mid 21)=\delta\left(\eta_{i}, 1\right) \delta\left(\eta_{j}, 2\right)+\delta\left(\eta_{i}, 2\right) \delta\left(\eta_{j}, 1\right),
$$

which is related to diffusion of prey between prey and predators;

$$
w_{\text {diff }}(20 \mid 02)=\delta\left(\eta_{i}, 2\right) \delta\left(\eta_{j}, 0\right)+\delta\left(\eta_{i}, 0\right) \delta\left(\eta_{j}, 2\right),
$$

which is related to diffusion of predators in empty sites.

ii) Reactions. The possible reaction processes are

$$
\begin{gathered}
X+Z \rightarrow X+X \\
X+Y \rightarrow Y+Y \\
Y \rightarrow Z
\end{gathered}
$$

and the cyclic process

$$
X \rightarrow Y \rightarrow Z \rightarrow X
$$

for the local reactions are always observed.

At each time step we choose a site $i$ at random and it is updated according to the following set of local rules:

(a) Prey birth. If site $i$ is empty then it can be occupied by a prey and the transition probability per site associated is

$$
w_{\text {react }}(1 \mid 0)=a \delta\left(\eta_{i}, 0\right) \frac{1}{z} \sum_{j} \delta\left(\eta_{j}, 1\right),
$$

where the summation is over the nearest neighbor of site $i, a$ is a parameter related to the prey birth probability, $z$ is the coordination number of lattice, $\delta(x, y)$ denotes here the Kronecker delta.

(b) Predation and predators birth. The process of predation is accompanied by the instantaneous death of prey and birth of predator. If the site $i$ is occupied by prey then in the next instant of time, with probability

$$
w_{\text {react }}(2 \mid 1)=b \delta\left(\eta_{i}, 1\right) \frac{1}{z} \sum_{j} \delta\left(\eta_{j}, 2\right),
$$

where $b$ is a parameter related to the predation probability, site $i$ will be occupied by a predator;

(c) Death of predators. The third reactive process is related to the death of predators, which happens spontaneously, with probability

$$
w_{\text {react }}(0 \mid 2)=c \delta\left(\eta_{i}, 2\right)
$$

where $c$ is a parameter related to the predators death. 


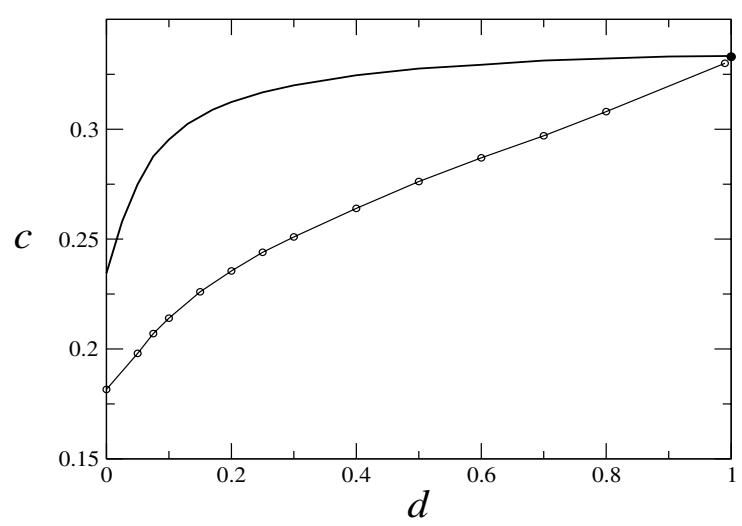

FIG. 1: Phase diagram in the plane $c$ versus $d$, obtained from pair mean-field (upper curve) approximation and from numerical simulations (lower curve), where $c$ is the predator death probability and $d$ is the diffusion probability. The probability $a$ of prey birth and the probability $b$ of predation are equal, $a=b=1-c$. The full circle corresponds to the transition point given by the simple mean-field approximation.

\section{DYNAMIC MEAN-FIELD APPROXIMATION}

We analyze the model by means of two levels of mean-field approximation: the simple (SMF) and pair (PMF) approximation $[39,52]$. In the SMF, the probability of a cluster of sites is approximated by a product of one-site probabilities. In this case it suffices to consider the time evolution for the density of prey $x=P(1)$ and the density of predator $y=P(2)$. The time evolution equations, obtained from the master equation, assuming that space is homogeneous and isotropic, are given by

$$
\frac{d x}{d t}=a x(1-x-y)-b x y
$$

and

$$
\frac{d y}{d t}=b x y-c y
$$

The solution of these equations gives a continuous phase transition from an active state $(0 \leq x \leq 1,0 \leq y \leq 1)$ to a prey absorbing state $(x=1, y=0)$ occurring at $b=c$. Here we are considering only the case $a=b$ which implies that the transition occurs at $c_{c r i t}^{S M F}=1 / 3[9,25]$. We point out that this approximation is not able to take into account explicitly the diffusion process so the resulting transition line does not depend on $d$. We note that equations (13) and (14) have the same form of equations (3) and (4) which were devised under the assumptions of a patch model.

PMF is the first approximation that is able to shown properties that depends explicitly on the diffusion. In this approximation correlations of higher order are written in terms of onesite and two-site probabilities. Assuming again that space is homogeneous and isotropic it suffices to use the two densities

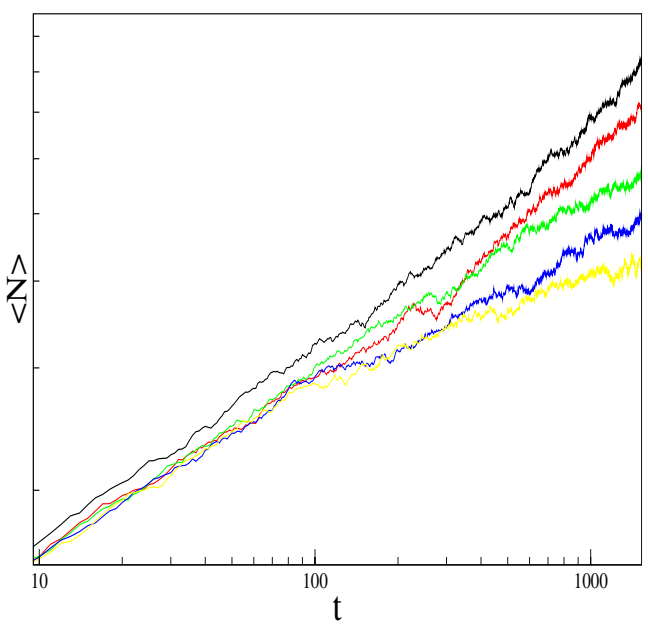

FIG. 2: Log-log plot of the time evolution of the mean number of predators $\langle N\rangle$. The figure shows the behavior of this quantity for $a=b, d=0.2$ and for five values of $c: 0.2348,0.2350,0.2354$, 0.2356 and 0.2358 , from top to bottom.

$x=P(1)$ and $y=P(2)$ and three nearest neighbor pair correlations $u=P(10), v=P(12)$ and $w=P(20)$. The time evolution equations for these variables are too cumbersome and will not be written down. These equations are integrated numerically by repeated iterations to get the stationary solutions. Figure 1 shows the transition line in the space $c$ versus $d$ for the case $a=b$. We see that as one increases the value of diffusion probability $d$ the transition from the active (where the species coexistence takes place) to the absorbing state occurs at higher values of $c$. That is, the diffusion process enhances the coexistence of species. We see that the transition line approaches the simple mean-field value, $c=1 / 3$, for sufficiently large diffusion, $d=1$.

\section{SIMULATIONAL RESULTS}

The simulation procedure is carried out as follows. At each time step we choose a random number $\zeta$ homogeneously distributed between 0 and 1 . If $\zeta<d$, the diffusion process is carried out. Otherwise the reactions of birth, death and predation are realized. That is, the diffusion, described by Eqs. (7), (8) and (9), occur with probability $d$ and the reaction processes (10), (11) and (12) occur with probabilities $(1-d) a$, $(1-d) b$ and $1-d) c$, respectively. We are also assuming that $a+b+c=1$.

We have performed time dependent simulations $[5,51]$ for the present model as follows. We have considered a regular square lattice and an initial condition where all sites of the lattice are completely covered by prey with the exception of the origin of the lattice which is occupied by one predator. With a set of samples we have analyzed some of the relevant quantities for the determination of the transition point. With this purpose we have studied the temporal behavior of the mean 


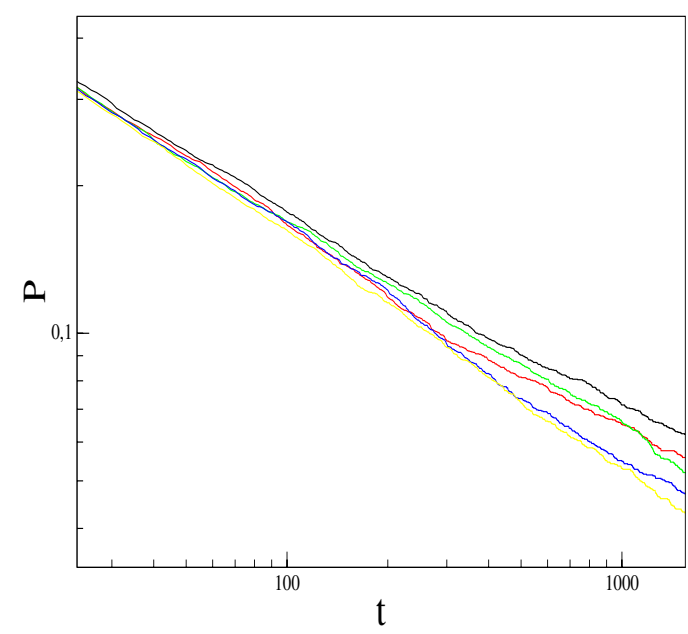

FIG. 3: Log-log plot of the time evolution of the survival probability $P$. The figure shows the behavior of this quantity for $a=b, d=$ 0.2 and for five values of $c: 0.2348,0.2350,0.2354,0.2356$ and 0.2358 , from top to bottom.

number of predators $\langle N\rangle$, the survival probability $P$, i.e. the probability of having at least one predator in the lattice at time $t$. We also have analyzed the mean-square distance of spreading of activity from the origin, $\left\langle R^{2}\right\rangle$, as a function of time. Each run is initiated by randomly chosen one site and them the rules defined in Eqs. (6)-(12) are applied. The updating is asynchronous and we consider lattice sizes sufficiently large so that predators do not reach the borders of the lattice during the simulation time.

According to the scaling laws for time dependent simulations [51] at criticality the following asymptotic behaviors are expected,

$$
\langle N\rangle \sim t^{\eta}, \quad P \sim t^{-\delta}, \quad\left\langle R^{2}\right\rangle \sim t^{z},
$$

where $\eta, \delta$ and $z$ are dynamic critical exponents.

Figures 2, 3 and 4 show the behavior of $\langle N\rangle, P(t)$ and $\left\langle R^{2}\right\rangle$ as a function of time for a given set of parameters $a, b$ and $d=0.2$ and different values of $c$ near criticality. According to the scaling relations (15) a straight line fitted to the data points in the log-log plot of these quantities allow us to obtain the localization of the critical point. Using the parametrization introduced in [9], [10], we can express the parameters $a, b$ and $c$, as $a=(1-c) / 2-p, b=(1-c) / 2+p$, with $-1 / 2 \leq p \leq 1 / 2$ and $0 \leq c \leq 1-2|p|$. For $p=0.0$ we estimate that the transition from the active state to the absorbing state, and diffusion probability $d=0.2$, occurs at $c_{c r i t}^{1}=0.2354$. For the same set of parameters, but without explicit diffusion (parameter $d=0.0$ ), it has been obtained $c_{c r i t}^{0}=0.1816(1)$ [9].

The exponent values were determined performing $100 \mathrm{sim}-$ ulations, each one with 5000 independent runs. Each simulation contributes with a value of the exponents in a histogram. The exponent values are estimated as the mean value of the associated distribution and the statistical errors are achieved

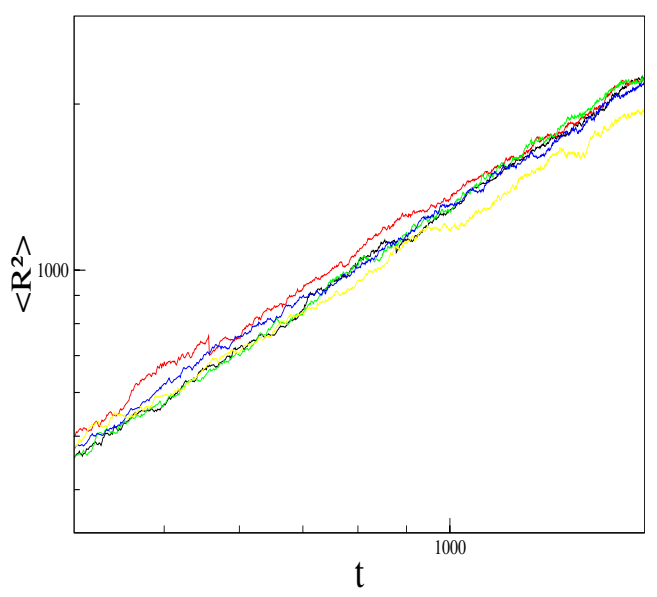

FIG. 4: Log-log plot of the time evolution of the mean square distance of spreading of predators $\left\langle R^{2}\right\rangle$. The figure shows the behavior of this quantity for $a=b, d=0.2$ and for five values of $c$ : $0.2348,0.2350,0.2354,0.2356$ and 0.2358 , from top to bottom.

through the standard deviations. As long as the histogram distribution is compatible with a Gaussian distribution, the use of this method can provide lower statistical errors with more simulations. If the histograms become incompatible with a Gaussian distribution then the errors can not be evaluated in such a way. In this case, the relevant error is the one associated to the critical value of $c$.

Considering this procedure we get the following estimates for the values of the dynamic critical exponents: $\eta=$ $0.236(5), \delta=0.441(8)$ and $z=1.129(8)$. Then, within the statistical errors, the values for the exponents $\eta, \delta$ and $z$, seem to be in agreement with the ones of the directed percolation universality class [5].

Finally, we observe that our numerical results show that, for the set of parameters considered here, the threshold of coexistence of species increases when the explicit diffusion process is considered.

\section{DISCUSSION}

We have performed a simulational analysis and also a mean-field analysis of a predator-prey stochastic structured model with diffusive process. The diffusion mimics the explicit movement of individuals in the habitat. We have considered that diffusion is allowed to occur, with a given probability, and for any pair of neighboring sites which are in different states. We have focused on the effect of these diffusive processes in the threshold of coexistence of species. For the set of parameters considered here our results indicate that local diffusion between any pair of neighboring elements (prey, predators and vacant sites) with the same probability, increases the threshold of species coexistence in the predatorprey stochastic lattice model. Our simulational results also indicate that, for the set of parameters considered, there is no modification in the critical behavior associated to the transi- 
tion from the active state (coexistence of species) to the prey absorbing state. The phase transition is continuous and seem to belong to the directed percolation universality class as it occurs for null diffusion.

It is worth noticing that the enlargement of the active phase is a consequence of the type of diffusive processes we have considered. The fact that prey can move over the lattice promotes their birth in empty sites and the meeting with predators. This gives place to the possible predation and birth of predators. Then the number of predators is increased even when their probability of death $c$ is considerably high. The movement of prey promotes the mixing of the space. For high values of diffusion the critical line given by the pair mean-field approximation approaches very quickly the simple mean-field result where the transition from the active state to the prey absorbing state occurs at $c_{c r i t}^{S M F}=1 / 3$ for $a=b$ (the set of parameters considered here).

In future work we plan to obtain the whole phase diagram of the model, as well as to analyze the possible types of species coexistence, for different values of the diffusion probability by means of extensive numerical simulations.

\section{Acknowledgment}

T. T. is obliged to J. A. Plascak, chairman of the V Brazilian Meeting on Simulational Physics, for the meeting invitation. The authors have been supported by the Brazilian agency CNPq.
[1] A. Lotka, J. Am. Chem. Soc. 42, 1595 (1920).

[2] A. Lotka,, Proc. Nat. Acad. of Sciences USA 6, 410 (1920).

[3] A. Lotka, Elements of Mathematical Biology (Dover, New York, 1924).

[4] V. Volterra, Leçons sur la Théorie de la Lutte pour la Vie (Ghautier-Villars, Paris, 1931).

[5] J. Marro and R. Dickman, Nonequilibrium Phase Transitions in Lattice Models (Cambridge University Press, Cambridge, 1999).

[6] T. Tomé e M. J. de Oliveira, Dinâmica Estocástica e Irreversibilidade (Editora da Universidade de São Paulo, São Paulo, 2001).

[7] R. Durrett and S. Levin, Theor. Popul. Biol. 46, 363 (1994).

[8] R. Durrett and S. Levin, J. Theor. Biol. 205, 201 (2000).

[9] J. E. Satulovsky and T. Tomé, Phys. Rev. E 49, 5073 (1994).

[10] K. C. de Carvalho and T. Tomé, Int. J. Mod. Phys. C 17, 1647 (2006).

[11] C. B. Huffaker, Hilgardia 27, 343 (1958).

[12] S. A. Levin, Am. Naturalist 108, 207 (1974).

[13] S. A. Levin and T. M. Powell (eds.), Patch Dynamics (Springer, New York, 1993).

[14] I. Hanski and M. E. Gilpin (eds.), Metapopulation Bilogy: Ecology, Genetics and Evolution (Academic Press, New York, 1997).

[15] A. Hastings, Theor. Popul. Biol. 12, 37 (1977)

[16] P. Grassberger, Math. Biosc. 63, 157 (1983).

[17] G. Nachman, J. Animal. Ecol. 56, 267 (1987).

[18] K. Tainaka, J. Phys. Soc. Japan, 57, 2588 (1988).

[19] A. M. de Roos, E. McCauley and W. G. Wilson, Proc. R. Soc. Lond. 246177 (1991).

[20] H. Matsuda, N. Ogita, A. Sasaki and K. Satô, Progress Theor Phys. 88, 1035, 1992.

[21] W. G. Wilson, A. M. de Roos and E. McCauley, Thoer. Popul. Biol. 43, 91 (1993).

[22] N. Boccara, O. Roblin and M. Roger, Phys. Rev. E 504531 (1994).

[23] L. Frachebourg, P. L. Krapivsky and E. Ben-Naim, Phys. Rev. E 54, 6186 (1996).

[24] D. Tilman and P. Kareiva, Spatial Ecology: The Rôle of Space in Populations and Interactions (Princeton University Press, Princeton, 1997).

[25] J. Satulovsky and T. Tomé, J. Math. Biol. 35, 344 (1997).

[26] J. Bascompte, R. V. Solé and N. Martinez, J. Theor. Biol. 187,
213 (1997)

[27] T. Antal and M. Droz, Phys. Rev. E 64, 036118 (2001).

[28] T. Antal, M. Droz, A. Lipowsky and G. Ódor, Phys. Rev. E 64 , 036118 (2001).

[29] O. Ovaskanien, K. Sato, J. Bascompte and I. Hanski, J. Theor. Biol. 215, 95 (2002)

[30] L. Berek, Ecol. Modell. 150, 55 (2002).

[31] K. C. de Carvalho and T. Tomé, in T. Tomé (ed.) Tendências da Física Estatística no Brasil (Editora Livraria da Física, São Paulo, 2003), p. 128

[32] G. Szabó and G. A. Sznaider, Phys. Rev. E 69, 031911 (2004).

[33] K. C. de Carvalho and T. Tomé, Mod. Phys. Lett. B 18, 873 (2004).

[34] M. A. M. de Aguiar, H. Sayama, M. Baranger, and Y. Bar-Yam, J. Stat. Phys. 114, 1417 (2004)

[35] D. Stauffer, A. Kunwar and D. Chowdhury, Physica A 352, 202 (2005).

[36] M. Mobilia, I. T. Georgiev and U. C. Tauber, Phys. Rev. E 73, 040903 (2006)

[37] I. Hanski and O. Ovaskainen, Nature 404, 755 (2006).

[38] E. Arashiro and T. Tomé, J. Phys. A 40, 887 (2007).

[39] K. C. de Carvalho and T. Tomé, Braz. J. Phys. 37, 466 (2007).

[40] T. Tomé and K. C. de Carvalho, J. Phys. A 40, 12901 (2007).

[41] S. B. L. de Araújo and M. A. M. de Aguiar, Phys. Rev. E 75, 061908 (2007).

[42] R. Levins, Bull. Entomol. Soc. Am. 15, 237 (1969).

[43] H. Haken, Synergetics, an Introduction (Springer, Berlin, 1976).

[44] G. Nicolis and I. Prigogine, Self-Organization in Nonequilibrium Systems (Wiley, New York, 1977).

[45] E. P. Odum, Fundamentals of Ecology (Saunders, Phyladelphia, 1959).

[46] J. D. Murray, Mathematical Biology (Springer, Berlin, 1989).

[47] E. Renshaw, Modelling Population Bilogy in Space and Time (Camdridge University Press, Cambridge, 1991).

[48] A. Hastings, Population Bilogy: Concepts and Models (Springer, New York, 1997).

[49] R. E. Riecklefs and G. L. Miller, Ecology (Freeman, New York, 2000).

[50] J. T. Tanner, Ecology 56, 855 (1975).

[51] P. Grassberger and A. de la Torre, Ann. Phys. 122, 373 (1979).

[52] R. Dickman, Phys. Rev. A 34, 4246 (1986). 